\title{
AN INVARIANCE OF DOMAIN RESULT FOR MULTI-VALUED MAXIMAL MONOTONE OPERATORS WHOSE DOMAINS DO NOT NECESSARILY CONTAIN ANY OPEN SETS
}

\author{
ATHANASSIOS G. KARTSATOS
}

(Communicated by Palle E. T. Jorgensen)

\begin{abstract}
Let $X$ be a real, reflexive, locally uniformly convex Banach space with $X^{*}$ locally uniformly convex. Let $T: X \supset D(T) \rightarrow 2^{X^{*}}$ be a maximal monotone operator and $G \subset X$ open and bounded. Assume that $M \subset X^{*}$ is pathwise connected and such that $T(D(T) \cap G) \cap M \neq \emptyset$ and $\overline{T(D(T) \cap \partial G)} \cap$ $M=\emptyset$. Then $M \subset T(D(T) \cap G)$. If, moreover, $T$ is of type $(S)$ on $\partial G$, then $\overline{T(D(T) \cap \partial G)}$ may be replaced above by $T(D(T) \cap \partial G)$. The significance of this result lies in the fact that it holds for multi-valued mappings $T$ which do not have to satisfy $\operatorname{int} D(T) \neq \emptyset$. It has also been used in this paper in order to establish a general "invariance of domain" result for maximal monotone operators, and may be applied to a greater variety of problems involving partial differential equations. No degree theory has been used. In addition to the above, necessary and sufficient conditions are given for the existence of a zero (in an open and bounded set $G$ ) of a completely continuous perturbation $T+C$ of a maximal monotone operator $T$ such that $T+C$ is locally monotone on $G$.
\end{abstract}

\section{INTRODUCTION AND PRELIMINARIES}

In what follows, the symbol $X$ stands for a real Banach space with norm $\|\cdot\|$ and (normalized) duality mapping $J$. Unless otherwise stated, we shall assume that $X$ is reflexive and that $X$ and $X^{*}$ have been renormed so that they are locally uniformly convex. This implies that the duality mapping $J$ is single-valued, monotone (see definition below) and bicontinuous.

The symbols $\left\langle x^{*}, x\right\rangle$ and $\left\langle x, x^{*}\right\rangle$ denote the value of the functional $x^{*} \in X^{*}$ at $x \in X$. We denote by $D(T), R(T)$ and $G(T)$ the effective domain, the range and the graph of a mapping $T: X \rightarrow 2^{X^{*}}$, respectively. We have $D(T)=\{x \in X: T x \neq \emptyset\}$ and $G(T)=\left\{\left(x, x^{*}\right): x \in D(T), x^{*} \in T x\right\}$. An operator $T: X \supset D(T) \rightarrow 2^{X^{*}}$ is said be "injective" on $G \subset X$ if $x, y \in D(T) \cap G$ and $T x \cap T y \neq \emptyset$ imply $x=y$. In what follows, "continuous" means "strongly continuous" and the symbol " $\rightarrow$ " (" - ") means strong (weak) convergence. The symbol $R\left(R_{+}\right)$stands for the set $(-\infty, \infty)([0, \infty))$ and the symbols $\partial D, \operatorname{int} D, \bar{D}$ denote the strong boundary, interior and closure of the set $D$, respectively. An operator $T: X \supset D(T) \rightarrow Y$,

Received by the editors September 12, 1995 and, in revised form, November 27, 1995.

1991 Mathematics Subject Classification. Primary 47H17; Secondary 47H05, 47H10.

Key words and phrases. Maximal monotone operator, pathwise connected set, invariance of domain, compact perturbation, existence of zeros. 
with $Y$ another real Banach space, is "bounded" if it maps bounded subsets of $D(T)$ onto bounded sets. It is "compact" if it is continuous and maps bounded subsets of $D(T)$ onto relatively compact sets. It is "completely continuous" if it is continuous from the weak topology of $D(T)$ to the strong topology of $Y$. It is called "demicontinuous" if it is continuous from the strong topology of $D(T)$ to the weak topology of $Y$.

An operator $T: X \supset D(T) \rightarrow 2^{X^{*}}$ is "monotone" if

$(*) \quad\left\langle u^{*}-v^{*}, x-y\right\rangle \geq 0$ for every $x, y \in D(T)$ and every $u^{*} \in T x, v^{*} \in T y$.

A monotone operator $T$ is "strongly monotone" if 0 in the right-hand side of $(*)$ is replaced by $\alpha\|x-y\|^{2}$, where $\alpha>0$ is a fixed constant. A monotone operator $T$ is called "maximal monotone" if its graph $G(T)$ is a maximal monotone subset of $X \times X^{*}$. A monotone operator $T$ is maximal monotone if and only if $R(T+\lambda J)=X^{*}$ for all $\lambda>0$. An operator $T: X \supset D(T) \rightarrow 2^{X^{*}}$ is said to be of "type $(S)$ " on the set $E \subset X$, if for every sequence $\left\{x_{n}\right\} \subset D(T) \cap E$ with $x_{n} \rightarrow x_{0} \in X$ and

$$
\lim _{n \rightarrow \infty}\left\langle v_{n}^{*}, x_{n}-x_{0}\right\rangle=0,
$$

for some $v_{n}^{*} \in T x_{n}$, we have $x_{n} \rightarrow x_{0}$.

We denote by $\operatorname{co} A$ the convex hull of a set $A$. Two sets $A \subset X, B \subset X$ are called "separated" if $(\bar{A} \cap B) \cup(A \cap \bar{B})=\emptyset$. A subset $M$ of a Banach space $X$ is called "pathwise connected" if for every $x, y \in M$ there exists a continuous function $s:[0,1] \rightarrow M$ such that $s(0)=x$ and $s(1)=y$. The function $s$ is called a "path." A pathwise connected set $M$, associated with the norm topology, is connected. A set $M$, associated with the norm topology, is pathwise connected if and only if it is connected and every point in $M$ has a pathwise connected neighborhood. For these facts, the reader is referred to Dugundji [5, pp. 114-116].

Let $G$ be an open and bounded subset of $X$. The purpose of this paper is to give a general theorem, Theorem 1, according to which a pathwise connected set $M \subset X^{*}$ lies in the range of a maximal monotone operator $T$. The conditions that the set $M$ has to satisfy are: $T(D(T) \cap G) \cap M \neq \emptyset$ and $\overline{T(D(T) \cap \partial G)} \cap$ $M=\emptyset$. The conclusion is: $M \subset T(D(T) \cap G)$. Results of this type were given recently by Guan [6] (for demicontinuous monotone operators with $G$ convex), Park [15] (for demicontinuous monotone operators in Hilbert spaces) and Kartsatos [13] (for demicontinuous monotone operators with $G$ not necessarily convex). The main characteristic of our result is the fact that $D(T)$ does not necessarily contain an open set, which results in the absence of any continuity property imposed upon the operator $T$. This result, Theorem 1, is then used in order to establish a result, Proposition 1, according to which a maximal monotone operator $T$ which is locally injective and of type $(S)$ on an open set $G$ has the property that $T(D(T) \cap G)$ is open. Finally, a result is given, Proposition 2, which says that the Leray-Schauder condition is necessary and sufficient for the existence of a zero of the sum $T+C$, where $T$ is maximal monotone and $C$ is completely continuous. We do require the additional assumption that $T+C$ is locally monotone on a bounded and open set $G$.

Lemma A. Let $\left\{s_{n}\right\}$ be a nonincreasing sequence of positive numbers, with $s_{n} \rightarrow 0$ as $n \rightarrow \infty$, and $\left\{x_{n}\right\} \subset X$. Assume that for all $m, n$ we have

$$
\left\langle s_{n} J x_{n}-s_{m} J x_{m}, x_{n}-x_{m}\right\rangle \leq 0 .
$$


Then $\left\{\left\|x_{n}\right\|\right\}$ is a nondecreasing sequence. If it is bounded, then $x_{n} \rightarrow$ (some) $x \in$ $X$.

This lemma follows easily from Lemma 1.4 of [2].

For facts about the theory of monotone operators the reader is referred to Barbu [1], Browder [3], Pascali and Sburlan [16] and Zeidler [19]. For results related to the ones in this paper, we refer to the papers [6]-[15] and some of the references therein.

\section{MAIN RESULTS}

Theorem 1. Let $T: X \supset D(T) \rightarrow 2^{X^{*}}$ be maximal monotone and let $G$ be an open and bounded subset of $X$. Assume that $M \subset X^{*}$ is pathwise connected and such that $T(D(T) \cap G) \cap M \neq \emptyset$ and $\overline{T(D(T) \cap \partial G)} \cap M=\emptyset$. Then $M \subset T(D(T) \cap G)$. If, moreover, $T$ is of type $(S)$ on $\partial G$, then $\overline{T(D(T) \cap \partial G)}$ can be replaced above by $T(D(T) \cap \partial G)$.

Proof. We may assume that $0 \in D(T) \cap G$ and $0 \in T(0)$; otherwise, for some fixed $y_{0} \in T(D(T) \cap G) \cap M$ and $x_{0} \in D(T) \cap G$ such that $y_{0} \in T x_{0}$, we replace the operator $T$ by the operator $\widetilde{T}(x) \equiv T\left(x+x_{0}\right)-y_{0}, x \in D(\widetilde{T})$, where $D(\widetilde{T}) \equiv$ $D(T)-x_{0}$. We also replace the sets $M, G$ by the sets $\widetilde{M} \equiv M-y_{0}$ and $\widetilde{G} \equiv G-x_{0}$, respectively. It is easy to see that $0 \in D(\widetilde{T}) \cap \widetilde{G}, 0 \in \widetilde{T}(0), 0 \in \widetilde{T}(D(\widetilde{T}) \cap \widetilde{G}) \cap \widetilde{M}, \widetilde{T}$ is maximal monotone on $D(\widetilde{T})$ and $\overline{\widetilde{T}}(D(\widetilde{T}) \cap \partial \widetilde{G}) \cap \widetilde{M}=\emptyset$.

Let $y^{*} \in M$ be fixed. Since $0 \in M$ and $M$ is pathwise connected, there exists a path $s:[0,1] \rightarrow M$ such that $s(0)=0$ and $s(1)=y^{*}$. Since $T$ is maximal monotone, the operator $T_{n} \equiv T+(1 / n) J$ is maximal monotone and surjective, for every $n=1,2, \ldots$. Thus, for each $n$ and $t \in[0,1]$, the inclusion

$$
T x+(1 / n) J x \ni s(t)
$$

is solvable. We show first that there exists a positive integer $n_{0}$ such that $T_{n}(D(T) \cap \partial G) \not \supset s(t)$, for any $n \geq n_{0}$ and any $t \in[0,1]$. To this end, assume that the contrary is true and let $\left\{n_{k}\right\}$, a subsequence of $\{n\}$, and $t_{k} \in[0,1], k=1,2, \ldots$, be such that $x_{n_{k}} \in D(T) \cap \partial G$ and

$$
T x_{n_{k}}+\left(1 / n_{k}\right) J x_{n_{k}} \ni s\left(t_{k}\right) .
$$

Since $[0,1]$ is compact, we may also assume that $t_{k} \rightarrow t_{0} \in[0,1]$. Since $G$ is bounded, there exists $y_{n_{k}}^{*} \in T x_{n_{k}}$ such that $y_{n_{k}}^{*} \rightarrow s\left(t_{0}\right)$. It follows that $s\left(t_{0}\right) \in$ $\overline{T(D(T) \cap \partial G)}$, i.e., a contradiction to our hypothesis on the set $M$. From this point on, we assume that $n \geq n_{0}$.

We now show that $y^{*} \in T_{n}(D(T) \cap G)$. The operator $T_{n}$ has a continuous singlevalued inverse $T_{n}^{-1}: X^{*} \rightarrow D(T)$. If we give $D(T)$ the relative topology induced on it by $X$, the mapping $T_{n}^{-1}: X^{*} \rightarrow D(T)$ is still continuous. Due to this important observation, its inverse image function takes a set open (closed) in $D(T)$ into a set which is open (closed) in $X^{*}$. Consequently, $T_{n}(D(T) \cap G)$ is open and $T_{n}(D(T) \cap \partial G)$ is closed in $X^{*}$. From

$$
\begin{aligned}
& T_{n}(D(T) \cap \bar{G})=T_{n}(D(T) \cap G) \cup T_{n}(D(T) \cap \partial G), \\
& \overline{T_{n}(D(T) \cap G)}=T_{n}(D(T) \cap G) \cup \partial T_{n}(D(T) \cap G)
\end{aligned}
$$


and

$$
\overline{T_{n}(D(T) \cap G)} \subset \overline{T_{n}(D(T) \cap \bar{G})}=T_{n}(D(T) \cap \bar{G}),
$$

it follows that

$$
\partial T_{n}(D(T) \cap G) \subset T_{n}(D(T) \cap \partial G) .
$$

Consequently, since the path $s$ does not have a point in common with the set $\partial T_{n}(D(T) \cap G)$, it must lie entirely in the set

$$
T_{n}(D(T) \cap G) \cup\left(\overline{T_{n}(D(T) \cap G)}\right)^{c},
$$

where $A^{c}$ denotes the complement of the set $A \subset X^{*}$. However, the sets

$$
T_{n}(D(T) \cap G),\left(\overline{T_{n}(D(T) \cap G)}\right)^{c}
$$

are separated. Since the range of $s$ is connected, being the continuous image of $[0,1]$, and $s(0) \in T_{n}(D(T) \cap G)$, we must have $s(t) \in T_{n}(D(T) \cap G)$, for every $t \in[0,1]$. Thus, $y^{*} \in T_{n}(D(T) \bigcap G)$.

Let $x_{n}$ denote the solution of

$$
T x+(1 / n) J x \ni y^{*} .
$$

Since $G$ is bounded, we may assume that, for some subsequence $\left\{x_{n_{k}}\right\}$ of $\left\{x_{n}\right\}$, we have $x_{n_{k}} \rightarrow x \in \overline{\operatorname{co} G}$. Then

$$
\left\langle\left(1 / n_{i}\right) J x_{n_{i}}-\left(1 / n_{j}\right) J x_{n_{j}}, x_{n_{i}}-x_{n_{j}}\right\rangle=-\left\langle v_{n_{i}}^{*}-v_{n_{j}}^{*}, x_{n_{i}}-x_{n_{j}}\right\rangle \leq 0,
$$

for all $i, j=1,2, \ldots$, where $v_{n_{i}}^{*} \in T x_{n_{i}}, v_{n_{j}}^{*} \in T x_{n_{j}}$. Lemma A implies now that $x_{n_{k}} \rightarrow x \in \bar{G}$. Since $v_{n_{k}}^{*} \rightarrow y^{*}$ and $T$ is maximal monotone, $x \in D(T)$ and $y^{*} \in T x$. Since $M \cap T(D(T) \cap \partial G)=\emptyset, x \in D(T) \cap G$.

Now, let $T$ be of type $(S)$ on $\partial G$. Then, in the argument above about $s\left(t_{0}\right) \in$ $\overline{T(D(T) \cap \partial G)}, x_{n_{k}} \rightarrow x_{0}$ and (2) imply

$$
\left\langle y_{n_{k}}^{*}, x_{n_{k}}-x_{0}\right\rangle=-\left\langle\left(1 / n_{k}\right) x_{n_{k}}, x_{n_{k}}-x_{0}\right\rangle+\left\langle s\left(t_{k}\right), x_{n_{k}}-x_{0}\right\rangle,
$$

for some $y_{n_{k}}^{*} \in T x_{n_{k}}$, and

$$
\lim _{k \rightarrow \infty}\left\langle y_{n_{k}}^{*}, x_{n_{k}}-x_{0}\right\rangle=0
$$

This implies that $x_{n_{k}} \rightarrow x_{0} \in \partial G$, and the maximality of $T$ yields $T x_{0} \ni s\left(t_{0}\right)$. Since $x_{0} \in D(T) \cap \partial G$, we have a contradiction to our assumption on the set $M$.

Before we establish the invariance of domain result for maximal monotone operators, Proposition 1, we need the following corollary to Theorem 1.

Corollary 1. Let $T: X \supset D(T) \rightarrow 2^{X^{*}}$ be maximal monotone and let $G$ be an open and bounded subset of $X$. Assume, further, that $T$ is of type $(S)$ on $\partial G$ and

$$
T(D(T) \cap G) \cap T(D(T) \cap \partial G)=\emptyset .
$$

Then $T(D(T) \cap G)$ is open.

Proof. Let $x_{0} \in D(T) \cap G$ and $y_{0} \in T x_{0}$. As in the proof of Theorem 1, we may assume that $x_{0}=0, y_{0}=0$. We show that there exists $r>0$ such that the open ball $B_{r}(0) \subset X^{*}$ lies in the set $T(D(T) \cap G)$.

We shall apply Theorem 1 with $M=B_{r}(0)$, for some $r>0$. We show first that there exists $r>0$ such that

$$
T(D(T) \cap \partial G) \cap B_{r}(0)=\emptyset .
$$


Let us assume that this is not true. Then there exists a sequence of positive numbers $\left\{r_{n}\right\}$ such that $r_{n} \rightarrow 0$ and

$$
T(D(T) \cap \partial G) \cap B_{r_{n}}(0) \neq \emptyset .
$$

This implies that there exists a sequence $\left\{x_{n}\right\} \subset D(T) \cap \partial G$ and a sequence $\left\{y_{n}^{*}\right\}$ such that $y_{n}^{*} \in T x_{n} \cap B_{r_{n}}(0)$ and $y_{n}^{*} \rightarrow 0$ as $n \rightarrow \infty$. Since $\left\{x_{n}\right\}$ is bounded, we may assume that $x_{n} \rightarrow x_{0} \in \overline{\operatorname{co} G}$. Then

$$
\lim _{n \rightarrow \infty}\left\langle y_{n}^{*}, x_{n}-x_{0}\right\rangle=0 .
$$

Since $T$ is of type $(S)$ on $\partial G, x_{n} \rightarrow x_{0} \in \partial G$ and, because $T$ is maximal monotone, $0 \in T x_{0}$ and $x_{0} \in D(T) \cap \partial G$. This implies $0 \in T(D(T) \cap G) \cap T(D(T) \cap \partial G)$, i.e., a contradiction to our assumption. Thus, for some $r>0,(3)$ is true. Obviously, $B_{r}(0)$ is pathwise connected. Thus, we can apply Theorem 1 to conclude that $B_{r}(0) \subset T(D(T) \cap G)$. The proof is complete.

In the next statement we do not assume that $G$ is bounded, but we do assume that $T$ is locally injective and locally of type $(S)$. Given an operator $T: X \supset$ $D(T) \rightarrow 2^{X^{*}}$, we say that $T$ has a property $\mathcal{P}$ "locally" on $G \subset X$ if for every $x_{0} \in D(T) \cap G$ there exists a ball $B_{r}\left(x_{0}\right) \subset G$ such that $T$ has the property $\mathcal{P}$ on $D(T) \cap B_{r}\left(x_{0}\right)$. If $G=X$, then we say simply that $T$ has "locally" the property $\mathcal{P}$.

Proposition 1. Let $T: X \supset D(T) \rightarrow 2^{X^{*}}$ be maximal monotone and let $G$ be an open subset of $X$. Then the following statements are true.

(i) If $T$ is locally injective and of type $(S)$ on $G$, then $T(D(T) \cap G)$ is open;

(ii) if $T$ is of type $(S)$, locally injective and $R(T)$ is closed in $X^{*}$, then $R(T)=X^{*}$.

Proof. (i) Since $T$ is locally injective and of type $(S)$ on $G$, given $x_{0} \in D(T) \cap$ $G$, there exists a ball $\overline{B_{q}\left(x_{0}\right)} \subset G$ such that $T$ is injective and of type $(S)$ on $\overline{B_{q}\left(x_{0}\right)}$. Since $\left(D(T) \cap B_{q}\left(x_{0}\right)\right) \cap\left(D(T) \cap \partial B_{q}\left(x_{0}\right)\right)=\emptyset, T\left(D(T) \cap B_{q}\left(x_{0}\right)\right) \cap$ $T\left(D(T) \cap \partial B_{q}\left(x_{0}\right)\right)=\emptyset$. By Corollary 1 , the set $T\left(D(T) \cap B_{q}\left(x_{0}\right)\right)$ is open. Since $T(D(T) \cap G)$ is a union of such sets, $T(D(T) \cap G)$ is also open.

(ii) By Part (i), for $G=X, R(T)=T(D(T) \cap X)$ is open. Since $R(T)$ is also closed, $R(T)=X^{*}$.

We now give a corollary to Theorem 1 which contains a criterion for the existence of a ball in the range of a maximal monotone operator $T$. For a set $A$ we set $|A| \equiv \inf \{\|u\|: u \in A\}$.

Corollary 2. Let $T: X \supset D(T) \rightarrow 2^{X^{*}}$ be maximal monotone and let $G$ be an open and bounded subset of $X$. If there exist $r>0, x_{0} \in D(T) \cap G$ such that

$$
\left|T x_{0}\right|<r \leq|T(D(T) \cap \partial G)|,
$$

then the ball $B_{r}(0) \subset X^{*}$ lies in the range of the operator $T$. Actually, we have $T(D(T) \cap G) \supset B_{r}(0)$.

Proof. Since

$$
|\overline{T(D(T) \cap \partial G)}| \geq r
$$

we have

$$
\overline{T(D(T) \cap \partial G)} \cap B_{r}(0)=\emptyset .
$$


Also, since $\left|T x_{0}\right|<r$, there exists $y_{0}^{*} \in T x_{0}$ such that $\left\|y_{0}^{*}\right\|<r$. Thus, $y_{0}^{*} \in B_{r}(0)$ and

$$
T(D(T) \cap G) \cap B_{r}(0) \neq \emptyset .
$$

Consequently, we can apply Theorem 1 with $M \equiv B_{r}(0)$. The proof is complete.

Yang proved Corollary 2 in [18] by using Browder's degree theory from [4].

We now give, for the first time, necessary and sufficient conditions for the existence of a zero of a completely continuous perturbation $C$ of a maximal monotone operator $T$. This is a rather interesting result, which shows the power of the LeraySchauder condition in the solvability of nonlinear inclusions involving some type of compactness or condensity. However, we do make the assumption that $T+C$ be locally monotone on an open and bounded set $G$.

Proposition 2. Let $T: X \supset D(T) \rightarrow 2^{X^{*}}$ be maximal monotone and $C: \overline{D(T)} \rightarrow$ $X^{*}$ completely continuous. Assume, further, that $G \subset X$ is open and bounded and such that $T+C$ is locally monotone on $G$. Then the following conditions are equivalent.

(a) $0 \in(T+C)(D(T) \cap G)$;

(b) there exist $r>0$ and $x_{0} \in D(T) \cap G$ such that $\overline{B_{r}\left(x_{0}\right)} \subset G$ and

$$
\left\langle u^{*}+C x, x-x_{0}\right\rangle \geq 0, \text { for every }\left(x, u^{*}\right) \in\left(D(T) \cap \partial B_{r}\left(x_{0}\right)\right) \times T x ;
$$

(c) there exist $r>0$ and $x_{0} \in D(T) \cap G$ such that $\overline{B_{r}\left(x_{0}\right)} \subset G$ and

$$
(T+C) x \not \supset \mu J\left(x-x_{0}\right) \text {, for every }(\mu, x) \in(-\infty, 0) \times\left(D(T) \cap \partial B_{r}\left(x_{0}\right)\right) .
$$

Proof. Assume that $0 \in(T+C)(D(T) \cap G)$. Then there exists $x_{0} \in D(T) \cap G$ such that $0 \in(T+C) x_{0}$. Since $T+C$ is locally monotone on $G$, there exists a ball $\overline{B_{r}\left(x_{0}\right)} \subset G$ such that $T+C$ is monotone on $D(T) \cap \overline{B_{r}\left(x_{0}\right)}$. Consequently,

$$
\left\langle u^{*}+C x, x-x_{0}\right\rangle \geq 0, \text { for every }\left(x, u^{*}\right) \in\left(D(T) \cap \partial B_{r}\left(x_{0}\right)\right) \times T x ;
$$

It follows that (a) $\Rightarrow$ (b).

To show that $(\mathrm{b}) \Rightarrow(\mathrm{c})$, assume that (b) holds and let $(T+C) x \ni \mu J\left(x-x_{0}\right)$, for some $(\mu, x) \in(-\infty, 0) \times\left(D(T) \cap \partial B_{r}\left(x_{0}\right)\right)$. Then, for some $u^{*} \in T x$,

$$
0 \leq\left\langle u^{*}+C x, x-x_{0}\right\rangle=\mu\left\langle J\left(x-x_{0}\right), x-x_{0}\right\rangle=\mu\left\|x-x_{0}\right\|^{2}<0 .
$$

This contradiction says that $(b) \Rightarrow(\mathrm{c})$.

Let (c) hold. We note that we may assume that $x_{0}=0$ and $0 \in T(0)$; otherwise we replace $T x$ by $\widetilde{T} x \equiv T\left(x+x_{0}\right)-v_{0}^{*}$ and $C x$ by $\widetilde{C} x \equiv C\left(x+x_{0}\right)+v_{0}^{*}$, where $x \in D(\widetilde{T}) \equiv D(T)-x_{0}$ and $v_{0}^{*} \in T\left(x_{0}\right)$. We also replace $G$ by $\widetilde{G} \equiv G-x_{0}$ and $B_{r}\left(x_{0}\right)$ by $B_{r}(0)$. Condition (4) would now read as follows:

$$
(\widetilde{T}+\widetilde{C}) x \not \supset \mu J x \text {, for every }(\mu, x) \in(-\infty, 0) \times\left(D(\widetilde{T}) \cap \partial B_{r}(0)\right) .
$$

We consider the approximate problem

$$
T x+C x+(1 / n) J x \ni 0 .
$$

We observe that since the operator $T$ is maximal monotone, the homotopy function

$$
H(t, x) \equiv x-(t T+(1 / n) J)^{-1}(-t C x),(t, x) \in[0,1] \times \overline{D(T)},
$$

is well-defined. In order to define $H(t, \cdot)$ on the set $\overline{B_{r}(0)}$, we restrict the operator $C$ on the closed and bounded set $\overline{D(T)} \cap \overline{B_{r}(0)}$. Since $X$ is reflexive, this restricted 
operator $C$ is compact. We extend $C$ to a compact operator on all of $X$ by Lemma 31 of Rothe [17]. We also use the symbol $C$ to denote this new compact operator. Now, we observe that the mapping $G_{t}: x \rightarrow(t T+(1 / n) J)^{-1}(-t C x)$ is compact for every $t \in[0,1]$. Also, $G_{t} x$ is continuous in $t$ uniformly w.r.t. $x \in \overline{B_{r}(0)}$ by the proofs of Theorems 3.2 and 3.7 of Guan and the author [7].

In order to be able to solve the equation $H(1, x)=0$ (hence (5)), with $x \in B_{r}(0)$, using the Leray-Schauder theory, it suffices to show that $H(t, x)=0$ has no solution $x_{t} \in \partial B_{r}(0)$, for any $t \in(0,1]$. To this end, let $x_{t} \in \partial B_{r}(0)$ solve $H(t, x)=0$, for some $t \in(0,1]$. Then $x_{t} \in D(T)$ and

$$
t(T+C) x_{t}+(1 / n) J x_{t} \ni 0,
$$

or

$$
T x_{t}+C x_{t} \ni-[1 /(n t)] J x_{t},
$$

which is a contradiction to (4). Let us call $x_{n}$ a solution of (5) lying in $B_{r}(0)$. We may assume that

$$
x_{n} \rightarrow \tilde{x} \in \overline{\operatorname{co}\left(D(T) \cap B_{r}(0)\right)} \subset \overline{\operatorname{co}\left(\overline{D(T)} \cap \overline{B_{r}(0)}\right)}=\overline{D(T)} \cap \overline{B_{r}(0)} .
$$

Here, we have used the fact that $\overline{D(T)}$ is convex (cf. Zeidler [19, p. 915]). Since $C$ is the original operator $C$ on the set $\overline{D(T)} \cap \overline{B_{r}(0)}$, it is completely continuous there and $C x_{n} \rightarrow C \tilde{x}$. Now, we may apply Property (b) in Zeidler [19, p. 915] to conclude that $\tilde{x} \in D(T)$ and $T \tilde{x}+C \tilde{x} \ni 0$. Since $\overline{B_{r}(0)} \subset G, \tilde{x} \in D(T) \cap G$.

We now consider another necessary and sufficient condition for the existence of a zero of a multi-valued maximal monotone operator $T$. Related conditions for demicontinuous monotone operators $T$ can be found in the papers by Guan [6] and the author [13].

Theorem 2. Let $T: X \supset D(T) \rightarrow 2^{X^{*}}$ be maximal monotone and let $G$ be an open, convex and bounded subset of $X$. Assume, further, that $T$ is of type $(S)$ on $\partial G$ and

$$
T(D(T) \cap G) \cap T(D(T) \cap \partial G)=\emptyset .
$$

Then the following are equivalent.

(i) $0 \in T(D(T) \cap G)$;

(ii) there exists $x_{0} \in D(T) \cap G$ such that $\left|T x_{0}\right| \leq|T x|$, for every $x \in D(T) \cap \partial G$.

Proof. It is obvious that (i) implies (ii). To show that (ii) implies (i), we observe first that Corollary 1 implies that the set $T(D(T) \cap G)$ is open in $X^{*}$. To show that $T(D(T) \cap \bar{G})$ is closed, let $\left\{y_{n}^{*}\right\} \subset T(D(T) \cap \bar{G})$ be such that $y_{n}^{*} \rightarrow y_{0}^{*} \in X^{*}$. Then there exists a sequence $x_{n_{k}} \subset D(T) \cap \bar{G}$ such that $x_{n_{k}} \rightarrow x_{0} \in \overline{D(T)} \cap \bar{G}$ and $y_{n_{k}}^{*} \in T x_{n_{k}}$. Invoking Problem 32.6 in Zeidler [19, p. 915], we have $x_{0} \in D(T)$ and $y_{0}^{*} \in T x_{0}$. Since $x_{0} \in D(T) \cap \bar{G}, T(D(T) \cap \bar{G})$ is closed. Since $T(D(T) \cap G) \cap$ $T(D(T) \cap \partial G)=\emptyset$, we use

$$
\begin{aligned}
T(D(T) \cap \bar{G}) & =T(D(T) \cap G) \cup T(D(T) \cap \partial G) \\
& \supset \overline{T(D(T) \cap G)} \\
& =T(D(T) \cap G) \cup \partial T(D(T) \cap G)
\end{aligned}
$$


to conclude that

$$
\partial T(D(T) \cap G) \subset T(D(T) \cap \partial G) .
$$

We are going to show that the set

$$
M=\cup\left\{t z^{*}: t \in[0,1]\right\}
$$

lies in the set $T(D(T) \cap G)$, where $z^{*} \in T x_{0}$ is chosen so that $\left\|z^{*}\right\|=\left|T x_{0}\right|$. This is possible due to the reflexivity and the local uniform convexity of the space $X^{*}$, and the fact that $T x_{0}$ is a closed and convex set (cf. Zeidler [19, p. 915]). To this end, we show first that $M \cap T(D(T) \cap \partial G)=\emptyset$. Let $y^{*} \in M \cap T(D(T) \cap \partial G)$. Then $y^{*}=t z^{*}$, for some $t \in[0,1]$, and $y^{*} \in T \bar{x}$, for some $\bar{x} \in D(T) \cap \partial G$. If $z^{*}=0$, we are done. Thus, we may assume that $z^{*} \neq 0$. If $y^{*}=0$, then $\left\|z^{*}\right\| \leq|T \bar{x}| \leq\left\|y^{*}\right\|=0$, i.e., a contradiction. It follows that $y^{*} \neq 0$, which says that there exists $t \in(0,1]$ such that $y^{*}=t z^{*}$. If $t=1$, then $y^{*} \in T(D(T) \cap G) \cap T(D(T) \cap \partial G)$, i.e., a contradiction. Thus, $t \in(0,1)$ and $t\left\|z^{*}\right\|<\left|T x_{0}\right| \leq|T \bar{x}| \leq\left\|y^{*}\right\|=t\left\|z^{*}\right\|$. This contradiction proves our assertion. Since the set $M$ is connected, $M \cap T(D(T) \cap G) \ni$ $z^{*}$ and $M \cap \partial T(D(T) \cap G)=\emptyset$, we must have $M \subset T(D(T) \cap G)$. The proof is complete.

\section{Discussion}

It does not seem possible to have an exact analogue of the proof Theorem 1 for $m$-accretive operators $T$, even in the case of uniformly convex $X$ and $X^{*}$, unless the duality mapping is weakly continuous in a neighborhood of zero and $T$ satisfies a certain type of closedness condition. Nevertheless, versions of Corollary 2 and a part of Proposition 2 exist for such operators in various papers in the references and some of the references therein. For example, the author has shown in [12] the following relevant result. The space $X$ is now a general Banach space.

Theorem A. Let $T: X \supset D(T) \rightarrow 2^{X}$ be m-accretive and $C: X \supset \bar{G} \rightarrow X$ compact, where $G$ is open and bounded. Assume that there exists $x_{0} \in D(T) \cap G$ such that

$$
T x+C x \not \supset \mu\left(x-x_{0}\right), \text { for every }(\mu, x) \in(-\infty, 0) \times(\partial G \cap D(T)) .
$$

Then $0 \in \overline{(T+C)(D(T) \cap G)}$. Moreover, $0 \in(T+C)(D(T) \cap G)$ under one of the following conditions:

(i) $X$ is uniformly convex, $G$ is convex and $C$ is completely continuous;

(ii) $T$ is strongly accretive on $D(T) \cap G$;

(iii) the compactness condition on $C$ is replaced by the following: $C$ is bounded, continuous and the resolvent $(T+I)^{-1}$ is compact.

Thus, a version of the sufficiency part of Part (iii) of Proposition 2 holds for $m$ accretive operators without the local accretivity condition on the set $G$. Actually, a theorem like Theorem A above holds for maximal monotone operators. We give this version below for the sake of completeness. We should also mention that necessary and sufficient conditions for the existence of a zero of a maximal monotone operator have been given by the author in [13], but the proofs there are considerably different from the ones herein. We assume below the usual properties of the spaces $X, X^{*}$ as in Section 2. 
Theorem 3. Let $T: X \supset D(T) \rightarrow 2^{X^{*}}$ be maximal monotone and $C: X \supset \bar{G} \rightarrow$ $X^{*}$ compact, where $G$ is open and bounded. Assume that there exists $x_{0} \in D(T) \cap G$ such that

$$
T x+C x \not \supset \mu J\left(x-x_{0}\right), \quad \text { for every }(\mu, x) \in(-\infty, 0) \times(\partial G \cap D(T)) .
$$

Then $0 \in \overline{(T+C)(D(T) \cap G)}$. Moreover, $0 \in(T+C)(D(T) \cap G)$ under one of the following conditions:

(i) $G$ is convex and $C$ is completely continuous;

(ii) $T$ is strongly monotone on $D(T) \cap G$;

(iii) the compactness condition on $C$ is replaced by the following: $C$ is bounded, continuous and the resolvent $(T+J)^{-1}$ is compact.

Proof. The proof is mainly a combination of the proof of Proposition 2 and the proof of Proposition 1 of [12]. It is therefore omitted.

\section{REFERENCES}

1. V. Barbu, Nonlinear semigroups and differential equations in Banach spaces, Noordhoff Int. Publ., Leyden (The Netherlands), 1975. MR 52:11666

2. H. Brézis, M. G. Crandall and A. Pazy, Perturbations of nonlinear maximal monotone sets in Banach space, Comm. Pure Appl Math. 23 (1970), 123-144. MR 41:2454

3. F. Browder, Nonlinear operators and nonlinear equations of evolution in Banach spaces, Proc. Symp. Pure Appl. Math., 18, Part 2, Providence, 1976.

4. F. Browder, Degree of mapping and its generalizations, Contemp. Math. 21 (1983), 15-40. MR 85e: 47086

5. J. Dugundji, Topology, Allyn \& Bacon, Boston, 1970. MR 33:1824

6. Z. Guan, Ranges of operators of monotone type in Banach spaces, J. Math. Anal. Appl. 174 (1993), 256-264. MR 95b:47068

7. Z. Guan and A. G. Kartsatos, Ranges of perturbed maximal monotone and m-accretive operators in Banach spaces, Trans. Amer. Math. Soc. 347 (1995), 2403-2435. MR 95i:47096

8. A. G. Kartsatos, Recent results involving compact perturbations and compact resolvents of accretive operators in Banach spaces, Proceedings of the First World Congress of Nonlinear Analysts, Tampa, Florida, 1992, vol. III, Walter De Gruyter, New York, 1995, pp. (2197-2222). CMP 96:12

9. A. G. Kartsatos, On compact perturbations and compact resolvents of nonlinear m-accretive operators in Banach spaces, Proc. Amer. Math. Soc. 119 (1993), 1189-1199. MR 94c:47076

10. A. G. Kartsatos, Sets in the ranges of sums for perturbations of nonlinear m-accretive operators in Banach spaces, Proc. Amer. Math. Soc. 123 (1995), 145-156. MR 95c:47072

11. A. G. Kartsatos, Sets in the ranges of nonlinear accretive operators in Banach spaces, Studia Math. 114 (1995), 261-273. CMP 95:14

12. A. G. Kartsatos, Degree theoretic solvability of inclusions involving perturbations of nonlinear $m$-accretive operators in Banach spaces, Yokohama Math. J. 42 (1994), 171-181. MR 96c: 47098

13. A. G. Kartsatos, On the connection between the existence of zeros and the asymptotic behaviour of resolvents of maximal monotone operators in reflexive Banach spaces, Trans. Amer. Math. Soc. (to appear).

14. A. G. Kartsatos, New results in the perturbation theory of maximal monotone and $m$-accretive operators, Trans. Amer. Math. Soc. 348 (1996), 1663-1707. MR 96j:47050

15. J. A. Park, Invariance of domain theorem for demicontinuous mappings of type $\left(S_{+}\right)$, Bull. Korean Math. Soc. 29 (1992), 81-87. MR 93d:47134

16. D. Pascali and S. Sburlan, Nonlinear mappings of monotone type, Sijthoff and Noordhoff Intern. Publ., Bucureşti, Romania, 1978.

17. E. H. Rothe, Introduction to Various Aspects of Degree Theory in Banach Spaces, Math. Surveys and Monographs, No. 23, A.M.S., Providence, 1986. MR 87m:47145 
18. G. H. Yang, The ranges of nonlinear mappings of monotone type, J. Math. Anal. Appl. 173 (1993), 165-172. MR 94b:47069

19. E. Zeidler, Nonlinear Functional Analysis and its Applications, II/B, Springer-Verlag, New York, 1990. MR 91b:47002

Department of Mathematics, University of South Florida, Tampa, Florida 33620-5700

E-mail address: hermes@gauss.math.usf.edu 